\section{NI VIVO NI MUERTO, SINO TODO LO CONTRARIO. REFLEXIONES SOBRE LA MUERTE CEREBRAL}

\author{
David Rodríguez-Arias \\ Instituto de Filosofía CCHS-CSIC \\ d.ra@csic.es
}

Cómo citar este artículo/Citation: Rodríguez-Arias, D. (2013). "Ni vivo ni muerto, sino todo lo contrario. Reflexiones sobre la muerte cerebral". Arbor, 189 (763): a067. doi: http://dx.doi. org/10.3989/arbor.2013.763n5004

Recibido: 13 julio 2012. Aceptado: 6 junio 2013.

RESUMEN: Determinar el momento exacto en que tiene lugar la muerte siempre ha sido un problema de difícil resolución. Las propuestas tradicionales y contemporáneas para justificar los criterios legales de determinación de la muerte se han visto condicionadas por intereses sociales - en particular, recientemente, relativos a los trasplantes de órganos-. Los criterios legalmente aceptados para declarar la muerte siguen careciendo de soporte conceptual o científico. En este contexto de ausencia de consenso sobre la demarcación exacta de la frontera entre la vida y la muerte, estas son algunas de las preguntas que abordo en este artículo: ¿Cómo podemos definir la muerte? ¿Es la determinación de la muerte una tarea basada únicamente en hechos, o también en juicios de valor? ¿Equivale la muerte cerebral a la muerte humana? ¿Qué papel le corresponde a la bioética en los debates relativos a la determinación de la muerte y la obtención de órganos?

PALABRAS CLAVE: Determinación de la muerte; Trasplantes de órganos; Gradualismo; Pluralismo; Definición de la muerte; Muerte cerebral; Bioética.

\section{NEITHER DEAD NOR ALIVE, BUT QUITE THE OPPOSITE. REFLECTIONS ON BRAIN DEATH}

Copyright: (C) 2013 CSIC. Este es un artículo de acceso abierto distribuido bajo los términos de la licencia Creative Commons Attribution-Non Commercial (by-nc) Spain 3.0.

ABSTRACT: Determining the precise moment of death has always been a difficult problem. Traditional and contemporary attempts to justify legally accepted standards for death determination have been based on social interests - particularly related, more recently, to organ transplantation policies. Legally accepted criteria for declaring death still lack both conceptual and scientific support. In this context of absence of consensus about the exact location of the boundary between life and death, this article aims to address the following issues: How can we define death? Is the task of determining death strictly based on facts, or do values also play a role? Should brain death be equated to death? What role should bioethics play in the debates related to death determination and organ procurement?

KEYWORDS: Death determination; Organ transplantation; Gradualism; Pluralism; Definition of death; Brain death; Bioethics. 


\section{INTRODUCCIÓN}

El lenguaje común y la ley asumen que vida y muerte son dos fenómenos claramente diferenciados y opuestos. A pesar de ello, muchos de quienes han observado la muerte de cerca describen que el límite que separa al existir del no existir no siempre está tan claro. Así como la vida no irrumpe de forma abrupta, la muerte tampoco es un suceso puntual, sino que tiene lugar tras un proceso gradual. En efecto, desde un punto de vista estrictamente biológico, la muerte tiene lugar en algún punto a lo largo de un continuo de degeneración celular.

En los seres humanos, como en la mayoría de los animales, el proceso degenerativo de muerte celular no comienza en la vejez, sino que tiene lugar desde el nacimiento. Por otro lado, las células humanas resisten la degradación por falta de oxígeno durante tiempos variables en función del tipo de que se trate, siendo posible trasplantar exitosamente córneas de un fallecido incluso siete días tras haber sido declarado muerto (Slettedal, Lyberg, Ramstad, Beraki and Nicolaissen, 2007). Tomarse en serio el carácter gradual del proceso de morir vuelve complicado identificar un momento en ese proceso degenerativo en el que un organismo dejaría de estar vivo para pasar a ser un cadáver. Algunos han querido sortear este problema sugiriendo que la muerte comienza cuando los procesos degenerativos superan en número o proporción a los generativos ${ }^{1}$. Sin embargo, lo más importante no parece ser cuándo alguien comienza a morirse, sino cuándo está (definitivamente) muerto.

\section{La muerte y el derecho}

"La muerte del individuo podrá certificarse tras la confirmación del cese irreversible de las funciones cardiorrespiratorias o del cese irreversible de las funciones encefálicas"

“[...]a efectos de la certificación de muerte y de la extracción de órganos, será exigible la existencia de un certificado médico firmado por tres médicos, entre los que debe figurar un Neurólogo o Neurocirujano y el Jefe de Servicio de la Unidad Médica donde se encuentre ingresado, o su sustituto" ${ }^{2}$.

La ley española, como la de la mayoría de los países, asume y alimenta una percepción abrupta del fenómeno de morir, al no reconocer ningún estatus vital intermedio entre la vida y la muerte ${ }^{3}$. Por otro lado, adjudica a la medicina un papel fundamental para identificar el momento de la muerte, asumiendo implícitamente que el diagnóstico de la muerte es una tarea objetiva que los expertos pueden realizar sin que les aparezcan dudas. Y en cierto sentido, la medicina tiene esa capacidad. En la mayoría de los casos, la capacidad de la medicina para determinar cuándo alguien está vivo y cuándo muerto no es puesta en cuestión. En otros, sin embargo, la demarcación de la frontera entre la vida y la muerte plantea dudas: así ocurre en el diagnóstico de muerte cerebral.

La muerte cerebral es una de las condiciones clínicas que la ley asimila a la muerte humana. Se corresponde con el cese irreversible de todas las funciones encefálicas (whole brain death). Con la ayuda de la tecnología de soporte vital, quienes se encuentran en muerte cerebral pueden mantener la respiración, el latido cardíaco y la temperatura corporal durante días, semanas, meses e incluso años (Shewmon, 1998). Se han descrito casos de mujeres embarazadas en muerte cerebral mantenidas hasta que el feto alcanzó un nivel de desarrollo suficiente para ser extraído con vida del útero de su madre, legalmente fallecida meses atrás (Lane et al., 2004; Nelson, 1994; Siegler and Wikler, 1982; Spike, 1999).

Una parte del público, al referirse a la muerte cerebral, no tiene nada claro que esos pacientes sean cadáveres (Herpin and Paterson, 2000; Siminoff, Burant and Youngner, 2004). En un conocido artículo publicado en 1989 se demostró que casi uno de cada tres profesionales estadounidenses involucrados en el cuidado de pacientes en muerte cerebral desconocía que están legalmente muertos (Youngner, Landefeld, Coulton, Juknialis and Leary, 1989).

¿Cómo interpretar estas dudas? ¿Constituyen un caso de ignorancia por parte de la población, susceptible de ser paliada con información? ¿O más bien se deben a que el concepto es en sí mismo confuso y el estatus vital de la muerte cerebral opinable?

La medicina puede determinar con objetividad cuándo un individuo presenta una pérdida total de sus funciones cerebrales, pero no tiene competencia para asimilar ese estado a la muerte humana. Para sostener que un individuo está muerto al haber perdido las funciones cerebrales - a pesar de conservar otras, como el latido cardíaco- sería preciso explicar por qué las funciones cerebrales tienen una importancia especial para la vida. Y esto último ningún argumento médico lo puede demostrar. Al asumir que la muerte cerebral equivale a la muerte, la ley ha tomado una decisión de significación $n^{4}$.

En este trabajo trataré de mostrar que, aunque a la población se le puede enseñar cuál es el estatus vital que las leyes de su país asignan a quienes se encuen- 
tran en muerte cerebral, no se le puede exigir que esté de acuerdo.

\section{ORIGEN DE LA MUERTE CEREBRAL}

La idea de "muerte cerebral" surgió a final de los años 60 como resultado del progreso en las técnicas de reanimación cardiopulmonar. La muerte cerebral nunca habría existido si los respiradores automáticos no se hubieran aplicado a pacientes con el cerebro severamente dañado por un traumatismo o un accidente cerebral. Puede resultar sorprendente que un progreso técnico haya conducido a una revisión de los criterios clásicos para diagnosticar la muerte humana. ¿Cómo fue esto posible?

Tradicionalmente, se consideraba que las únicas funciones responsables de la vida eran la circulación y la respiración. El cese circulatorio acarreaba irremediablemente el cese respiratorio y viceversa. Cualquiera de esas pérdidas era irreversible y significaba la muerte. Además, cuando las medidas de reanimación todavía no se aplicaban, las personas que padecían un daño cerebral severo automáticamente perdían sus funciones cardiorrespiratorias. Eso hizo que el funcionamiento del corazón y los pulmones pareciera estar necesariamente ligado al funcionamiento del cerebro. Los métodos de reanimación cardiorrespiratoria vinieron a demostrar que eso no era cierto. Por un lado, gracias a la reanimación cardiaca se demostró que muchos paros cardíacos podían revertirse. Por otro lado, gracias a la respiración mecánica, la función respiratoria podía mantenerse artificialmente en pacientes con el cerebro destruido. El resultado fue la aparición de pacientes irreversiblemente inconscientes y con el organismo funcionando (gracias al mantenimiento asistido de la función respiratoria).

A principios de 1959, Wertheimer, Jouvet y Descortes describieron por primera vez este estado y lo caracterizaron como "muerte del sistema nervioso" (Escalante, 1996). Ese mismo año, dos neurólogos franceses describieron 23 casos de lo que ellos conceptualizaron como "coma dépassé". Definieron este diagnóstico en los siguientes términos:

"[E]s el coma en el que, a la abolición total de las funciones de la vida de relación, no de las perturbaciones, se añade una abolición total de las funciones de la vida vegetativa" (Mollaret and Goulon, 1959, 4).

Aunque estos autores llegaron a plantearse la dificultad de determinar "las fronteras últimas de la vida", su discurso todavía asimilaba ese estado a una forma de supervivencia. Estimaban que los casos de pacientes mantenidos con un respirador pero irreversiblemente inconscientes constituían un triste precio a pagar (rançon) por la capacidad adquirida para reanimar a ciertos pacientes que en condiciones normales habrían muerto:

\begin{abstract}
"Un precio - señalaban-, pues la supervivencia en el coma dépassé impone esfuerzos crecientes a los equipos de cuidados intensivos y prolonga un espectáculo cada vez más doloroso para la familia" (Mollaret and

Goulon, 1959, 4).
\end{abstract}

Los autores no soslayaron la cuestión sobre si se debía permitir morir a esos pacientes. Pero rechazaron esa posibilidad. A finales de los años 60 , la limitación del esfuerzo terapéutico no era una práctica común al ser asimilada a una eutanasia. Esta es probablemente la razón por la que Mollaret y Goulon reconocieron "no haber podido, ni querido, consentir [para este tipo de pacientes] el gesto del pollice verso"5.

\section{LOS TRASPLANTES DE ÓRGANOS}

La aparición de los primeros pacientes en coma dépassé coincide históricamente con el desarrollo de los primeros trasplantes de órganos. Los trasplantes de riñón se llevaban haciendo desde hacía años, pero con donantes vivos o con cadáveres declarados muertos de acuerdo con el criterio clásico -cardiorrespiratorio- de la muerte. Los órganos procedentes de estos cadáveres presentaban el inconveniente de su deterioro, producido por la pérdida de riego sanguíneo tras el paro circulatorio. La isquemia ${ }^{6}$ provocaba rechazos del órgano trasplantado en el receptor, lo que hacía que los índices de supervivencia de los receptores se contaran en horas, días, o como mucho, semanas.

En junio de 1963, el médico belga Guy Alexandre tuvo la audacia de extraer un riñón de un paciente en coma depassé, antes de que su corazón dejara de latir, para trasplantarlo en otro paciente con insuficiencia renal. Su intención era reducir el tiempo de isquemia y evitar el rechazo. Y en gran medida lo consiguió. El receptor de aquel riñón vivió casi tres meses (Machado, 2005). En 1966, durante un congreso de trasplantes, Alexandre manifestó haber practicado para entonces 9 extracciones de riñón de pacientes en coma dépassé. Algunos afamados trasplantadores, como Thomas Starlz, expresaron sus dudas sobre la licitud de ese procedimiento: "Dudo que alguno de los miembros de nuestro equipo de trasplantes pueda aceptar que una persona esté muerta si su corazón sigue latiendo" (Machado, 2005, 1940). Starlz estaba planteando si es aceptable emplear a pacientes vivos 
como fuentes de órganos para trasplante. ¿Es lícito extraer órganos de un paciente vivo? Esta cuestión se planteó en toda su radicalidad cuando se obtuvieron los primeros éxitos en los trasplantes de órganos impares (necesarios para la vida), como el corazón. ¿Es lícito causar la muerte al extraer órganos? El primer trasplante exitoso de corazón lo practicó el doctor Christian Barnard en Sudáfrica en 1967. El corazón procedía de Denise Ann Darvall, una mujer que había sufrido un traumatismo craneal durante un accidente de tráfico en Ciudad del Cabo. El propio Barnard certificó su muerte, aparentemente después de que tuviera lugar una parada cardiaca, tras interrumpirse el respirador automático. El receptor de aquel corazón, Louis Washkansky, vivió durante dieciocho días. Este caso testimoniaba algo extremadamente paradójico: ¿cómo podía ser que el corazón por cuya parada había muerto Darvall pudiera salvar la vida de otra persona?

La esperanza generada por el caso no acalló ciertas voces de preocupación ante la eventualidad de que se estuvieran sacrificando prematuramente algunas vidas para el beneficio de terceros (Lock, 2002, 82-3). Cada vez parecía más claro que el desarrollo exitoso de las políticas de trasplante iba a depender de la confianza social en los trasplantes de órganos y en el sistema médico.

En 1968, una comisión de expertos a la que se le encomendó la tarea de redefinir la muerte humana, estimó que la confianza del público solo podría preservarse evitando la sospecha de que a los donantes se les mataba al serles extraídos sus órganos. Esto es lo que más tarde se ha denominado la regla del donante fallecido (dead donor rule) que establece que no se debe matar al extraer órganos, o que solo se pueden extraer órganos vitales de cadáveres (Robertson, 1999).

\section{EL COMITÉ AD HOC PARA LA MUERTE CEREBRAL DE HARVARD}

Los pacientes en coma dépassé planteaban a finales de los años 60 dos problemas. Por un lado, a pesar de que nunca recobrarían la conciencia, estaban siendo los destinatarios de unos recursos caros y limitados. Por otro, no podían ser donantes de órganos impares, - como el corazón - sin que a los cirujanos extractores se les pudiera acusar de homicidio. El comité de expertos reunido en la Escuela de Medicina de Harvard para redefinir la muerte encontró una solución para no malgastar las camas de cuidados intensivos, promover la donación de órganos de pacientes en coma dépassé y disolver simultáneamente las preocupaciones sociales con respecto a la integridad profesional de los cirujanos extractores. La solución del Comité Ad Hoc para la muerte cerebral de Harvard consistía en considerar que los pacientes en coma dépassé ya estaban muertos. Llamando al coma dépassé "muerte cerebral" y asimilando esta a la muerte humana, se favorecía la limitación del esfuerzo terapéutico eludiendo el debate sobre la eutanasia, y se "evitaban controversias al extraer órganos" de aquellos pacientes que eran candidatos ideales para la donación, al no violarse la regla del donante fallecido (no se mata a quien ya está muerto).

Con el paso del tiempo, la "redefinición de la muerte" operada en Harvard ha resultado ser extremadamente eficaz como estrategia para promover la donación de órganos y la limitación del esfuerzo terapéutico en pacientes irreversiblemente inconscientes. Pero ha creado nuevos problemas, tanto teóricos como prácticos.

Defenderé a continuación que gran parte de los problemas que plantea la muerte cerebral tienen su origen en la falta de distinción entre dos cuestiones de índole diversa: 1. ¿Cuándo está muerta una persona? y 2. ¿Cuándo es éticamente aceptable realizar una extracción de órganos? La primera cuestión tiene en principio más que ver con consideraciones fácticas (con juicios de hecho) que la segunda, que es una cuestión eminentemente normativa (tiene que ver, fundamentalmente, con juicios de valor). De todas formas, se verá que la distinción misma entre hechos y valores es en sí misma muy problemática. Lo que de momento me interesa señalar es el modo en que el Comité Ad Hoc de Harvard presentó una cuestión genuinamente moral bajo el aspecto de una cuestión meramente fáctica. Al hacerlo, el Comité de Harvard escamoteó el debate sobre cuestiones bioéticas relacionadas con la asignación de camas de cuidados críticos, la limitación del esfuerzo terapéutico y la regla del donante fallecido.

Algunos se han preguntado hasta qué punto es válida la estrategia de redefinir un problema para resolverlo. (Singer, 1997) ¿En qué medida las necesidades prácticas de liberar camas de hospitales y obtener órganos para trasplantes son el tipo adecuado de razón para decidir sobre el estatus vital de pacientes? El Comité para la muerte cerebral de Harvard no ofreció ninguna razón de tipo científico para asimilar la muerte cerebral a la muerte. Su principal motivación la constituían las ventajas de racionalizar unos recursos sanitarios escasos - no desperdiciar órganos que podían salvar vidas si eran trasplantados - sin generar controversia social (Ad Hoc Committee of the Harvard Medical School to Examine the Definition of Brain Death, 1968). ¿Es esta una justificación aceptable? 
EN BUSCA DE UNA JUSTIFICACIÓN: EL MODELO DEFINICIÓN, CRITERIOS, TESTS

Hasta 1981, no se ofreció un argumento científico a favor del criterio de muerte cerebral. Tal argumento llegó de la mano de un neurólogo, un psiquiatra y un filósofo, cuya contribución sigue constituyendo el principal apoyo teórico de la asimilación legal de muerte cerebral a la muerte (Bernat, 1999; Bernat, Culver and Gert, 1981). En 1981, J. Bernat, Ch. Culver y B. Gert se propusieron paliar el déficit de legitimación científica de la muerte cerebral a través de una definición de la muerte. Poco más tarde, una comisión presidencial norteamericana a la que se le encargó analizar los problemas relacionados con la definición de la muerte y ofrecer una definición unitaria para una ley federal asumió las conclusiones de Bernat y sus colaboradores (1981). La propuesta señalaba la necesidad de distinguir tres aspectos en toda formulación sobre la muerte humana: una definición de la muerte, unos criterios para identificarla, y unas pruebas para determinarla empíricamente. Bernat, Culver y Gert consideraban fundamental establecer, en primer lugar, una definición con la que volver explícito el significado de la muerte. Entendían que esa es una labor fundamentalmente filosófica, aunque creían que esa definición debía aproximarse al concepto "común" u ordinario de la muerte?:

"Creemos que una comprensión adecuada del significado ordinario de la palabra o del concepto de muerte debe desarrollarse antes de que se elija un criterio. Debemos decidir qué es lo que comúnmente se entiende por muerte antes de que los médicos puedan decidir cómo medirla" (389).

En segundo, lugar, y como deducción lógica de la definición escogida, entendían que es preciso determinar unos criterios operativos para identificar en la práctica la muerte, tal y como había sido definida. Elegir unos criterios - consideraron Bernat, Culver y Gert-, es una tarea principalmente médica. En tercer y último lugar, una vez que se dispone de unos criterios de la muerte, había que elegir unos tests o pruebas clínicas o instrumentales que indiquen cuándo el criterio se ha satisfecho.

La Comisión Presidencial para la Determinación de la Muerte consideró: 1 . Que la muerte podía definirse como la pérdida del funcionamiento integrado del organismo como conjunto; 2. Que los criterios en los que tal definición se cumple son, o bien la pérdida irreversible de las funciones de todo el cerebro, o bien la pérdida irreversible de las funciones cardiorrespiratorias; y 3. Que las pruebas para constatar los dos criterios anteriores son aquellas que permiten evidenciar una pérdida irreversible de las funciones cardíacas (electrocardiograma plano, etc.) o una pérdida total e irreversible de las funciones del cerebro (electroencefalograma plano, ausencia de reflejos del troncoencéfalo, evidencia de falta de riego al cerebro.... ${ }^{8}$.

\section{LA TAREA DE DEFINIR: ASPECTOS LINGÜÍSTICOS}

El modelo definición, criterios, tests avalado por la President's Commission se apoya en una concepción clásica (aristotélica) del lenguaje, de acuerdo con la cual los conceptos cumplen la función de captar la esencia de los objetos o, lo que es lo mismo, su diferencia específica (Chiong, 2005). Parte de un presupuesto metafísico esencialista: todos los individuos que caen bajo un mismo concepto tienen en común algo que los identifica entre sí y los distingue de los demás individuos. El modelo podría representarse gráficamente del siguiente modo:

Suponiendo que los individuos " $A$ ", " $B$ ", " $C$ " y " $D$ " están muertos, la esencia de la muerte quedaría constituida por las características que todos tienen en común y que solo los muertos presentan. La definición debería recoger esas características y solo esas.

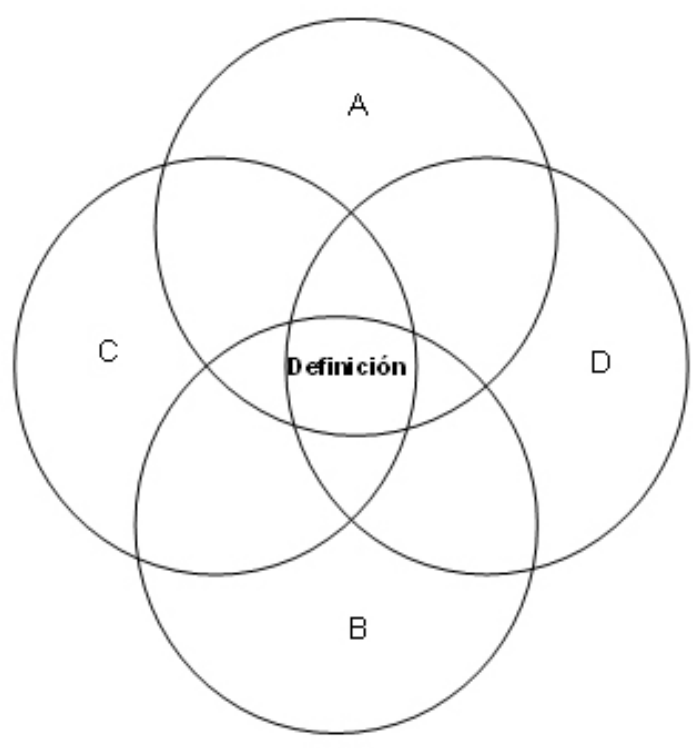

\section{EL MODELO OFICIAL}

La propuesta de la President's Commission implica que la muerte humana puede determinarse siguiendo un doble estándar (criterio): la muerte cerebral (o whole brain death) o la muerte cardiorrespiratoria (Capron, 1999). Excluye de la categoría de "muertos" 
a los pacientes que tienen una pérdida parcial de las funciones del cerebro, como los pacientes en estado vegetativo permanente. Este enfoque, pese haber sido asumido por las legislaciones de casi todos los países del mundo, ha recibido dos tipos de objeciones. Según algunos autores, es demasiado restrictiva, ya que otros pacientes, como los pacientes en estado vegetativo o los bebés anencefálicos, también deberían considerarse muertos (Veatch, 1999; Gervais, 1986). Estos autores tienden a pensar que no es necesario que muera todo el cerebro para considerar que una persona está muerta: bastaría una pérdida irreversible de las funciones corticales, responsables de la conciencia y de la cognición, que son, para muchos, las capacidades específicas del ser humano (este criterio ha venido a conocerse como higher brain death) (Green and Wikler, 1980). Otros estiman que el doble estándar es excesivamente laxo, pues solo el cese irreversible de la circulación indica una pérdida del funcionamiento integrado del organismo como conjunto (Shewmon, 2001).

El criterio cardiorrespiratorio, como criterio suficiente de muerte humana, plantea el problema de determinar su irreversibilidad (tras varios minutos de paro circulatorio, se dan casos de pacientes que recuperan el ritmo cardiaco), y levanta sospechas al plantear como posibilidad que se declare muerto a alguien sin haber objetivado una pérdida total de sus funciones cerebrales (Bernat, 2010; Bernat, 2013; Munjal et al., 2013; Rodriguez-Arias and Deballon, 2012). Estas objeciones desafían la oportunidad del criterio cardiorrespiratorio por dos motivos: 1 . casi nadie está dispuesto a admitir la posibilidad de la resucitación (la muerte se asume como un estado irreversible) y 2. nadie aceptaría que alguien está muerto si puede conservar alguna forma, por precaria que sea, de conciencia (Youngner, Arnold and DeVita, 1999). Por otro lado, el criterio de muerte cerebral ha sido cuestionado, no solo por parecer a muchos contraintuitivo (el cuerpo de pacientes en muerte cerebral está caliente y su corazón sigue latiendo) (Siminoff, Burant, 2004), sino por haberse demostrado que el cerebro no juega un papel tan determinante en el funcionamiento del organismo como se creyó durante buena parte del siglo XX (Shewmon, 2001). Estas constataciones enfrentan a los partidarios de la muerte cerebral a un dilema: o bien tienen que radicalizar su criterio (exigir que, además de las funciones cerebrales, se haya perdido toda actividad cerebral), o bien deben abandonar su pretensión de justificar la muerte encefálica en nombre del supuesto papel regulador del cerebro en el funcionamiento del organismo como conjunto.
Para sortear todas estas dificultades, los partidarios del criterio higher brain han propuesto abandonar la pretensión de hacer basar el diagnóstico en una concepción biológica de la muerte - lo que implica considerar que la muerte de los seres humanos no coincide con la pérdida de sus funciones orgánicas, sino con la pérdida de las capacidades que son más representativas de su esencia: la conciencia y la cognición, la personeidad (personhood) (Gervais, 1986) o la identidad personal (personal identity) (Gomila, 1999; Green and Wikler, 1980). Esta propuesta - que nuevamente asume el planteamiento esencialista del que parten Bernat et al. - conduce a considerar muertos a todos los pacientes con pérdidas del funcionamiento del cerebro lo suficientemente graves como para haber desaparecido las capacidades necesarias para el comportamiento humano, lo que incluiría, también, a los pacientes en estado vegetativo permanente y a los bebés anencefálicos. Tal vez la mayor objeción a esta propuesta es que, si la asimilación de la muerte cerebral a la muerte ya resulta contraintuitiva, más lo resultaría la idea de la muerte cortical, en la que no solo hay un mantenimiento espontáneo del latido cardiaco, sino también de la respiración.

\section{CUESTIONES SOBRE EL ESTATUTO EPISTEMOLÓGICO DE LA MUERTE CEREBRAL. NATURALISTAS VS. NOR- MATIVISTAS}

Las dificultades que genera la determinación de la muerte tienen su origen en cuestiones - todavía irresueltas - relativas al estatuto ontológico y epistemológico de la muerte. Me refiero a cuestiones cuyas respuestas aclararían a qué tipo de entidad se refiere uno cuando afirma de alguien que ha muerto (de quién o de qué se dice que muere) o cómo estar seguro de que así ha sucedido (quién tiene autoridad para constatar la muerte y por qué medios): ¿Qué tipo de entidad es la que muere, un organismo o una perso$n a$ ? ¿Determinar la muerte es una forma de constatar un hecho, o equivale a emitir un juicio de valor? ¿Es la muerte un fenómeno natural, o una construcción social? ¿Sobre qué bases debe proponerse una definición de la muerte? ¿Sobre bases biológicas? ¿Éticas? ¿Metafísicas? Si los médicos no tienen capacidad para asimilar la muerte cerebral a la muerte humana, ¿quién tiene autoridad para hacerlo?

Una posible clasificación de las propuestas de definición de la muerte humana es la que las separa entre naturalistas y normativistas. La tesis defendida por quienes comprenden la muerte humana desde un enfoque naturalista es que $a$ los pacientes en muerte 
cerebral se los considera como muertos porque están muertos. Entienden que la muerte es un hecho biológico cuya constatación nada tiene que ver con valoraciones ni con decisiones humanas. Quienes, como James Bernat, Culver y Gert, abrazan esta concepción, estiman que lo que muere es un organismo y no una entidad metafísica, como la persona. La presencia o ausencia de la capacidad de integración, entiende Bernat, es algo objetivo y no sujeto a decisiones: simplemente se constata. Frente a esta opción se sitúan los defensores de una concepción normativista de la muerte (Veatch, 1999). Para ellos, los pacientes en muerte cerebral están muertos porque se los considera muertos, y no al revés. La muerte es en este caso entendida como una construcción social dependiente de valores, no como un hecho biológico. Está muerto aquel que en un momento y lugar determinados está socialmente aceptado como tal. Es decir, es la sociedad, y no la naturaleza, quien decide cuándo alguien está vivo y cuándo deja de estarlo. Analicemos con más detalle cada una de estas concepciones, así como las respectivas críticas que han recibido.

\section{LA MUERTE COMO HECHO OBJETIVO: EL ENFOQUE NATURALISTA}

El argumento de Bernat, Culver y Gert y asumido por la Pressident's Commission constituye un caso del enfoque naturalista. Su planteamiento puede resumirse en cuatro puntos:

1. Presupuesto teórico: la muerte es la pérdida definitiva del funcionamiento integrado del organismo en su conjunto.

2. Afirmación empírica: el cerebro es el órgano regulador del funcionamiento de todo el organismo.

3. Pronóstico: Los pacientes en muerte cerebral dependen del respirador automático pero aunque este se le mantenga, necesariamente terminan por padecer un colapso cardio-respiratorio en un plazo breve.

4. Conclusión: La pérdida de las funciones cerebrales equivale a la pérdida de la vida.

A este planteamiento biológico se le han dirigido objeciones que denuncian su incoherencia interna. Partiendo del mismo presupuesto naturalista y biológico, estas objeciones acaban demostrando la inconsistencia entre la definición de la muerte y los criterios que Bernat y sus colaboradores han propuesto para determinarla. Las críticas señalan: 1 . que ciertas funciones integradoras no radican en el cerebro ${ }^{10} ; 2$. que el cerebro de pacientes correctamente diagnosticados en muerte cerebral conserva funciones integradoras $^{11}$; y 3 . que los pacientes en muerte cerebral puede llegar a "sobrevivir" durante varios años si se les sigue manteniendo conectados a un respirador automático ${ }^{12}$. Estas objeciones enfrentan a Bernat y los partidarios de la justificación oficial de la muerte cerebral a un dilema: o abandonan su definición de la muerte, o aceptan que los criterios para determinarla se vuelvan más exigentes (hasta el punto de que muchos de los pacientes actualmente diagnosticados en muerte cerebral deberían ser considerados como vivos). La alternativa explorada por los autores normativistas, consiste en abandonar la pretensión de definir la muerte desde presupuestos biológicos.

\section{LA CONSTRUCCIÓN SOCIAL DE LA MUERTE: EL ENFO- QUE NORMATIVISTA}

La medicina puede demostrar cuándo un cerebro ha dejado de estar irrigado y también cuándo un corazón ha dejado de tener actividad. Puede inferir a partir de esas constataciones cuándo el cerebro de un paciente haya dejado de funcionar en su totalidad. Sin embargo, no puede deducir de ello que ese paciente ha muerto. Para dar ese paso lógico es preciso adoptar lo que Karen Gervais denomina una decisión de significación. Puesto que en el cuerpo de pacientes en muerte cerebral sigue manteniéndose mucha actividad metabólica, la ciencia debería explicar por qué las funciones del cerebro son esenciales para la vida y no otras que se mantienen en el organismo de esos pacientes. La respuesta de Bernat, Culver y Gert, es que el cerebro se encarga de la integración del resto del organismo. Sin embargo, como se ha visto, esa tentativa de hacer consistir la vida en la capacidad de integración, y en justificar el criterio de la muerte cerebral en esa definición, ha fracasado. Y no por motivos metafísicos o morales, sino por motivos científicos: los pacientes en muerte cerebral siguen teniendo un funcionamiento integrado. Se sigue de esto que, si la ciencia desea asimilar la muerte cerebral a la muerte puede hacerlo, pero para ello deberá recurrir a presupuestos extracientíficos.

A la inconsistencia conceptual cabe añadir el hecho de que ese concepto naturalista no es expresión de la idea que toda la sociedad tiene de la muerte. La definición de la vida propuesta por la medicina no es compartida por quienes entienden que estar muerto para una persona significa haber perdido la conciencia y la cognición, o para quienes creen que se está muerto al haber perdido la identidad personal, o para quienes creen que lo que marca el paso de la vida a la muerte 
es la separación del alma con respecto al cuerpo. Si no puede demostrarse la verdad de ninguna de estas definiciones de la muerte; si en toda definición de la muerte intervienen creencias filosóficas y valores culturales y religiosos; si la asimilación de la muerte cerebral a la muerte carece de fundamento científico, ¿por qué la mayoría de las leyes establecen sin dejar lugar a ninguna objeción que la muerte cerebral equivale a la muerte? ¿Por qué una definición, de tantas posibles, se ha impuesto sobre las demás? ¿Por qué debería imponerse?

Robert Veatch y Alireeza Bagheri (Bagheri, 2007; Veatch, 1999) han defendido que las leyes deberían reconocer a las personas un derecho a elegir, entre ciertas opciones, la definición de la muerte que se les debería aplicar. Entienden que, puesto que existen varias definiciones posibles de la muerte y ninguna ha demostrado ser más válida que las demás, ninguna definición "oficial" de la muerte debería imponerse contra la autonomía de los individuos. En una sociedad pluralista, estiman, las personas deben poder elegir qué definición se les debe aplicar en caso de encontrarse en algún estado fronterizo entre la vida y la muerte. Bagheri ofrece el ejemplo de Japón, como el país que más cerca está de aceptar este pluralismo ${ }^{13}$.

\section{HECHOS, VALORES Y PLURALISMO}

En una sociedad pluralista existe una presunción a favor de respetar la autonomía de las personas y se necesitan razones - generalmente de orden público- para no hacerlo. Muchas decisiones que se toman en la relación clínica comportan una dimensión normativa, lo que obliga a considerar, no ya solo los aspectos técnicos relativos a la dimensión biológica de una patología, sino también la dimensión subjetiva o biográfica del paciente. Reconocer que varias visiones sobre lo que es correcto o incorrecto pueden ser igualmente válidas tiene sentido dado el carácter genuinamente irreconciliable de las diferentes propuestas de fundamentar lo que es bueno y lo que es correcto (Rorty, 2000). Por supuesto, pueden aducirse razones pragmáticas y de interés común para rechazar el pluralismo en algunos casos, pero de forma general este se reconoce en aquellas cuestiones que son de carácter genuinamente moral, como la de si se debe o no interrumpir un respirador automático o si se debe o no proceder a extraer los órganos de un individuo ${ }^{14}$. Ahora bien, la decisión sobre si alguien está vivo o muerto ¿acaso forma parte de este tipo de decisiones? Estar vivo o muerto: ¿hasta qué punto se trata de una cuestión normativa y en qué medida es un asunto fáctico? El enfrentamiento entre posturas naturalistas y posturas normativistas da cuenta de lo controvertido de este punto.

Con independencia de la comprensión conceptual que se tenga de la muerte, aceptar que las personas puedan decidir cuándo están vivas o muertas de acuerdo a sus propios valores plantea algunos riesgos. Si el criterio último para decidir cuándo alguien está vivo o muerto es su propia creencia sobre lo que significa estar vivo o muerto, entonces las personas que disfrutan típicamente de una perfecta salud física y psíquica podrían acabar teniendo derecho a que se las considerase muertas. En sentido contrario, algunos podrían llegar a exigir de forma anticipada que, cuando su cuerpo se encontrase en estado de descomposición, se le siguieran reconociendo los derechos de que disfrutan los vivos, incluido el de recibir tratamientos y cuidados. Bagheri es consciente del riesgo de relativismo que comporta su propuesta, por lo que restringe su pluralismo "a las opciones socialmente aceptadas". Desgraciadamente, no ofrece muchos detalles sobre lo que entiende por "socialmente aceptado" (Molina, Rodríguez-Arias and Youngner, 2008). Esto hace que su argumento para escapar al riesgo de relativismo resulte débil. Por un lado, si lo "socialmente aceptado" es el criterio determinante para establecer qué condiciones son asimilables a la muerte y cuáles no, la sociedad nunca podría equivocarse al considerar a un vivo como muerto. Por otro, ese razonamiento conduce a consecuencias claramente contraintuitivas: si una sociedad lo aceptase, un paciente con demencia pero consciente y con buena calidad de vida aparente podría ser considerada muerta por el simple hecho de que lo hubiera pedido mientras era competente. En contra de lo que sugiere Bagheri, lo socialmente aceptado no equivale necesariamente a lo éticamente aceptable.

\section{SUPERANDO LA DICOTOMÍA ENTRE HECHOS Y VALORES}

De lo dicho hasta ahora se sigue que tanto las propuestas naturalistas como las normativistas son controvertidas. A la propuesta naturalista le falta consistencia conceptual por no ser capaz de engranar con coherencia la definición que enuncia, los criterios aceptados para explicitar esa definición, y las pruebas exigidas para identificar en la práctica el cumplimiento de los criterios. Por otro lado, las propuestas normativistas dan lugar a consecuencias teóricas y prácticas inaceptables.

Refiriéndose a otro concepto borroso, el de "enfermedad", Peter Schwartz sostiene que la clasificación 
entre definiciones naturalistas y normativistas resulta poco operativa, al no permitir categorizar aquellas definiciones para las que lo patológico puede depender simultáneamente de consideraciones fácticas y valorativas (Schwartz, 2007). Entre quienes han teorizado sobre la muerte, algunos como Youngner y Arnold han planteado precisamente que se trata de un fenómeno con un pie en la naturaleza y otro en la cultura (Youngner and Arnold, 2001). ¿Cuál es exactamente la relación entre hechos y valores, cuál la implicación de la naturaleza y de la cultura en la determinación de la muerte humana? ¿Hasta qué punto la muerte se descubre, y hasta qué punto se decide cuándo alguien ha muerto?

Comúnmente se asume que hechos y valores son dos cosas dicotómicas: los hechos son objetivos, los valores subjetivos; los hechos se constantan, sobre los valores se opina. Hilary Putnam ${ }^{15}$ ha argumentado en contra de la creencia de que existe una dicotomía absoluta entre hechos y valores. También niega la conclusión que toda la tradición empirista, desde Hume hasta Carnap, deduce de ella, a saber, que los enunciados morales no son verdaderos ni falsos ${ }^{16}$.

Al principio he sugerido que el éxito del informe del Comité Ad Hoc para la Muerte cerebral de Harvard consistió en haber encubierto estratégicamente una serie de preguntas genuinamente morales (si es o no éticamente aceptable dejar morir a personas en coma dépassé al interrumpir el respirador automático y si es éticamente aceptable causar su muerte durante la extracción de sus órganos para trasplante), presentándolas como $-\mathrm{y}$ reduciéndolas $\mathrm{a}-$ una cuestión de hecho ("el coma dépassé es la muerte"). Al señalar esto no pretendo insinuar que hechos y valores sean absolutamente independientes. Muy a menudo, los asuntos normativos dependen de circunstancias fácticas. Por ejemplo, el hecho de que durante los 10 primeros días después de la concepción el embrión humano pueda convertirse en dos gemelos (y que por lo tanto todavía no se puede decir que sea un individuo) es relevante en una discusión sobre si es o no éticamente correcto emplear ciertos métodos anticonceptivos, como la píldora postcoital ${ }^{17}$. En otro sentido, si rechazo moralmente el racismo es (al menos en parte) porque sé que no existe ninguna superioridad racial entre las personas pertenecientes a distintos grupos étnicos. De manera que los juicios morales no están completamente desvinculados de enunciados descriptivos, y estos pueden condicionar a aquellos. $\mathrm{Si}$ no son independientes, ¿ puede decirse que los juicios morales son más objetivos de lo que habitualmente suele entenderse? H. Putnam defiende una forma de cognitivismo moral, puesto que cree que los enunciados morales pueden ser verdaderos o falsos ${ }^{18}$. La noción putnamiana de conceptos éticos densos resulta útil para comprender hasta qué punto el lenguaje puede ser simultáneamente descriptivo y normativo. Expresiones como "cruel", "impertinente", "sensible", "insensible", "humillante", "grosero", "celoso", "amable", "obstinado" o "molesto" son instancias de conceptos éticos densos. Todas ellas tienen esa doble lectura: Si mi pareja me califica como alguien "celoso", puede ser que esté juzgando mi comportamiento, y también puede ser que lo esté describiendo.

A pesar de que no exista ningún hecho empíricamente constatable que señale el momento de la muerte humana, tampoco parece que su determinación sea un juicio de valor puro. Si en el diagnóstico de la muerte incurren simultáneamente constataciones y valoraciones, tal vez tenga sentido decir que "muerto" es otro ejemplo - insólito quizá- de concepto ético denso.

\section{EL ABANDONO DE LA DEFINICIÓN}

La mayoría de quienes se han preocupado por el fundamento de la muerte cerebral han creído que en la secuencia definición-criterios-tests estaba la clave para resolver los problemas de justificación de la muerte cerebral. Al hacerlo, han aceptado por completo el modelo clásico y esencialista del lenguaje que opera a través de definiciones en términos de condiciones necesarias y suficientes ${ }^{19}$.

Una minoría de autores entiende, sin embargo, que los problemas de coherencia entre la definición, los criterios y las pruebas no pueden resolverse cambiando de definición ni afinando los criterios y los tests requeridos para que aquella se cumpla (Stoecker, 2012). Creen más bien que esas dificultades tienen precisamente su origen en la pretensión de resolver el problema de la justificación a través de una definición ${ }^{20}$. Por ello, consideran oportuno abandonar por completo el modelo que Chiong ha dado en llamar la letanía definición-criterios-tests (Chiong, 2005). Estos autores adoptan una concepción wittgensteiniana del lenguaje, alternativa a la clásica, de acuerdo con la cual el significado de un concepto no capta unos rasgos comunes a todos los objetos que constituyen la referencia de ese concepto. Wittgenstein señala que la mayoría de los conceptos al uso, como el de "silla", permiten a las personas entenderse a pesar de carecer de una definición clara y compartida de lo que es una silla (Wittgenstein, 2004). 
La aportación de Wittgenstein consiste en señalar cómo, para ser operativo, al lenguaje le basta con relacionar objetos a través de simples "parecidos de familia". Los parecidos de familia no son rasgos que todos y cada uno de los miembros de una familia de objetos compartan. Piénsese en el concepto de "silla": hay sillas con cuatro patas, pero también con tres, con una pata, e incluso sin patas. Los parecidos de familia no son por lo tanto rasgos "esenciales" (necesarios), sino características contingentes. Por supuesto, en toda familia de objetos hay individuos que presentan todos o la mayoría de los atributos característicos de esa familia: son los casos centrales o paradigmáticos. Pero también hay individuos que solo comparten algunos parecidos de familia y que son, por lo tanto, casos periféricos. Cuando son muy pocos los rasgos que comparte un individuo con el caso paradigmáti- co, podemos estar ante un caso fronterizo. La muerte cerebral y el estado vegetativo permanente pueden verse como casos fronterizos entre la vida y la muerte.

La concepción wittgensteiniana del lenguaje puede explicarse a través de la metáfora del racimo (cluster). Los individuos de una misma familia o racimo de individuos comparten algunas propiedades. Sin embargo, no hay ninguna propiedad que todos los miembros del racimo comparan. El hecho de tener alguna propiedad o parecido de familia simplemente hace que un individuo del racimo tenga más probabilidades de compartir otras propiedades con otros individuos del mismo racimo o familia. Esta es la razón de que los miembros de una misma familia se ejemplifican recíprocamente, pero de un modo que no es necesario (tampoco arbitrario), sino contingente. El modelo podría representarse gráficamente del siguiente modo:

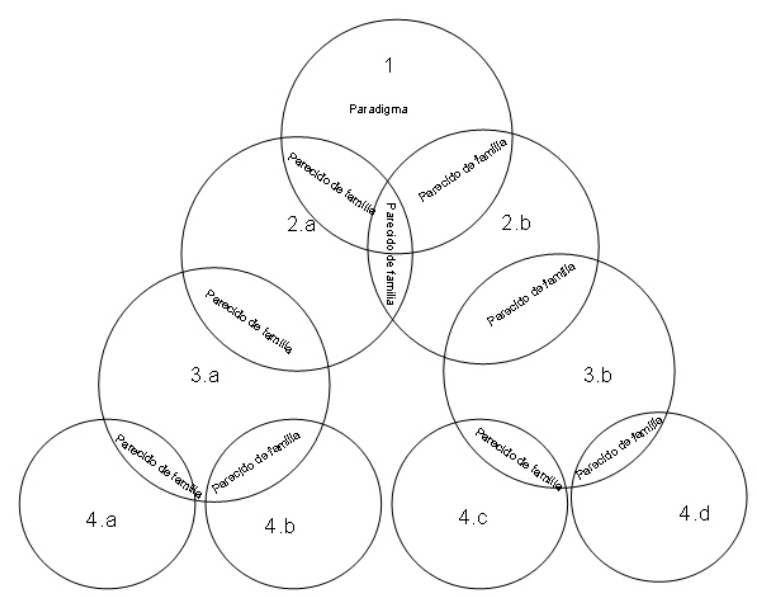

Piénsese en la familia García Sánchez. Julio y María han tenido dos hijos: Roberto y Alberto. A su vez, Alberto ha tenido otros dos hijos, Lucía y Jorge, (nietos de Julio y María). Obsérvense los rasgos fisonómicos de cada uno de sus miembros:

\begin{tabular}{|c|c|c|c|c|c|c|c|}
\hline & ojos claros & tez clara & pelo rizado & $\begin{array}{c}\text { frente } \\
\text { despejada }\end{array}$ & nariz chata & boca grande & $\begin{array}{c}\text { orejas de } \\
\text { soplillo }\end{array}$ \\
\hline Julio García & $\checkmark$ & $\checkmark$ & $\checkmark$ & $\checkmark$ & $\checkmark$ & $\checkmark$ & $\checkmark$ \\
\hline $\begin{array}{c}\text { María } \\
\text { Sánchez }\end{array}$ & & $\checkmark$ & $\checkmark$ & $\checkmark$ & $\checkmark$ & $\checkmark$ & $\checkmark$ \\
\hline Roberto & $\checkmark$ & $\checkmark$ & $\checkmark$ & $\checkmark$ & & $\checkmark$ \\
\hline $\begin{array}{c}\text { Alberto (padre } \\
\text { de Ly J) }\end{array}$ & $\checkmark$ & $\checkmark$ & $\checkmark$ & $\checkmark$ & $\checkmark$ & $\checkmark$ & $\checkmark$ \\
\hline Lucía & & $\checkmark$ & & & $\checkmark$ & & \\
\hline Jorge & $\checkmark$ & & & & \\
\hline
\end{tabular}


Muchas características fisonómicas son predominantes en el grupo. Sin embargo, no hay ni una sola característica que todos compartan. Los hermanos Jorge y Lucía no comparten ni un solo rasgo. ¿Acaso impide eso categorizarlos como hermanos? En absoluto: bastará conocer a su padre para reconocer su parentesco. Jorge no tiene la nariz chata - rasgo que presentan los demás miembros de su familia-. ¿Acaso suscita eso la sospecha de que Alberto no sea su padre biológico? En absoluto. Antes bien, los amigos de la familia le repetirán "icómo has salido a tu padre!" o "tienes los mismos ojos que tu abuelo paterno". Algo similar le sucede a otras familias de objetos, como las que reúnen conceptos como "droga"21 o "secta"22: aparte de los casos centrales o paradigmáticos que claramente ejemplifican la idea al poseer todas o la mayoría de las características típicas de esa familia de objetos, hay casos limítrofes que no comparten con el paradigma ni una sola característica pero sí con algunos parientes del paradigma, lo que los hacen merecedores de la misma denominación.

El concepto de "muerte" podría pensarse de este modo: no existe ninguna condición (ni el funcionamiento integrado del organismo como conjunto, ni la cognición, ni la identidad personal...) cuya pérdida implique necesaria y suficientemente la muerte. Tan solo hay condiciones cuya presencia en un individuo le hace más susceptible de pertenecer a la familia de los muertos. El caso paradigmático presentaría todas ellas: pérdida de la conciencia, pérdida de la respiración espontánea, pérdida del latido espontáneo, pérdida del funcionamiento integrado, pérdida de la capacidad de reproducción, pérdida de la temperatura corporal, pérdida de la capacidad para resistir a la entropía... Un cadáver en descomposición sería el paradigma de la muerte, mientras que un niño jugando durante la hora del recreo podría ser un paradigma de vida. Entre ambos casos, hay individuos que participan en diferentes grados de la idea de muerte: un individuo en muerte cerebral, un paciente en parada cardiaca, un paciente con una insuficiencia respiratoria crónica, un individuo en estado vegetativo permanente, un individuo con síndrome de locked-i $n^{23}$, un varón estéril... Según Chiong, no es necesario tener todos los rasgos típicos de los cadáveres para ser considerado como muerto. Esto justificaría por qué la muerte cerebral, a pesar de ser un estado compatible con la capacidad de reproducción, con la conservación de algunas funciones integradoras, con el latido espontáneo, etc. se asimila a la muerte.

\begin{tabular}{|c|c|c|c|c|c|c|}
\hline & Conciencia & $\begin{array}{c}\text { Respiración } \\
\text { espontánea }\end{array}$ & $\begin{array}{c}\text { Latido } \\
\text { espontáneo }\end{array}$ & $\begin{array}{c}\text { Funcionamiento } \\
\text { Integrado }\end{array}$ & $\begin{array}{c}\text { Capacidad } \\
\text { Reproductiva }\end{array}$ & $\begin{array}{c}\text { Resististencia } \\
\text { a la entropía }\end{array}$ \\
\hline $\begin{array}{c}\text { Cadáver en } \\
\text { Descomposición }\end{array}$ & & & $\checkmark$ & $\checkmark$ & $\checkmark$ \\
\hline $\begin{array}{c}\text { Parada cardiaca } \\
\text { Irreversible }\end{array}$ & $\checkmark$ & $\checkmark$ & $\checkmark$ & $\checkmark$ & $\checkmark$ \\
\hline $\begin{array}{c}\text { Muerte } \\
\text { Cerebral }\end{array}$ & $\begin{array}{c}\text { Estado } \\
\text { Vegetativo } \\
\text { Permanente }\end{array}$ & $\checkmark$ & $\checkmark$ & $\checkmark$ & $\checkmark$ & $\checkmark$ \\
\hline $\begin{array}{c}\text { Insuficiencia } \\
\text { respiratoria } \\
\text { crónica }\end{array}$ & $\checkmark$ & $\checkmark$ & $\checkmark$ & $\checkmark$ & $\checkmark$ & $\checkmark$ \\
\hline Locked-in & $\checkmark$ & $\checkmark$ & $\checkmark$ & $\checkmark$ & & $\checkmark$ \\
\hline Varón estéril & $\checkmark$ & $\checkmark$ & $\checkmark$ & $\checkmark$ & $\checkmark$ \\
\hline
\end{tabular}




\section{LOS PACIENTES EN MUERTE CEREBRAL NO ESTÁN MUERTOS PERO DEBE PERMITIRSE LA EXTRACCIÓN DE SUS ÓRGANOS}

Me gustaría terminar este artículo exponiendo mi propia propuesta para afrontar los problemas relativos a la definición de la muerte. Consiste en desvincular claramente la cuestión sobre el estatus vital de la muerte cerebral de la cuestión sobre la licitud de la extracción de órganos en ese tipo de pacientes, para discutir abiertamente la regla del donante fallecido. Gomila recuerda que somos los humanos, no la naturaleza, quienes necesitamos saber cuándo un paciente está vivo y cuándo muerto (Gomila, 1999). Una razón por la que las sociedades quieren estar al tanto cuanto antes del momento en el que un paciente deja de estar vivo es que la muerte ha marcado históricamente el comienzo de toda una serie de prácticas sociales, como el duelo, las autopsias, la inhumación o, más recientemente, la extracción de órganos. Prácticas que las intuiciones morales más comunes impedirían realizar si se creyera que el paciente sigue estando vivo. Resulta interesante que, en el caso de la muerte cerebral, no ocurre que el diagnóstico legal de la muerte marque el pistoletazo de salida para que comience la mayoría de esas prácticas. Casi todas se realizan solo después de que se le interrumpe el respirador automático al individuo y su actividad cardiaca cesa. Por ejemplo, nadie entierra a un paciente en muerte cerebral mientras su corazón late y conectado a un respirador... La extracción de órganos constituye en este sentido una excepción: comienza tan pronto como se certifica la muerte y se obtiene la autorización de la familia. La necesidad de extraer los órganos mientras están siendo perfundidos por el corazón hace que no se pueda esperar hasta la parada cardiaca. Esta fue una de las razones que llevaron a la Comisión de Harvard a proponer un criterio de muerte que fuera compatible con la permanencia del ritmo cardíaco. Otra razón fue la necesidad de interrumpir los respiradores automáticos para liberar las camas de esos pacientes y evitar medidas fútiles sin que los médicos pudieran ser acusados de causar la muerte de sus pacientes (Arnold and Youngner, 1993). Afortunadamente, las leyes han evolucionado desde los años 70 hasta permitir que los tratamientos fútiles puedan ser interrumpidos en los pacientes antes de que mueran: ya no es necesario que alguien esté muerto para que sea lícito interrumpirle un respirador automático.

Un mismo tipo de razonamiento puede conducir a la siguiente interrogante: ¿acaso es necesario considerar que un paciente en muerte cerebral está muerto para que sea legítimo extraerle sus órganos?
Asimilar legalmente la muerte cerebral a la muerte puede servir para que quienes consideran que siempre es ilícito causar la muerte secunden las extracciones de órganos de los pacientes con el cerebro irreversiblemente destruido. Llamar "muerto" a un paciente en muerte cerebral es una forma eficaz de encubrir el propósito utilitarista que impulsa a toda política de trasplantes de órganos. La asimilación de la muerte encefálica a la "muerte" ha conseguido escamotear el debate bioético sobre la regla del donante fallecido. Pero llamar "muerto" a un individuo en muerte cerebral no ha servido ni para proteger a los pacientes, ni para respetar su autonomía personal. Menos aún para fomentar un debate público en el que las diferentes concepciones de la muerte que articula la sociedad puedan ser escuchadas. Quienes han propuesto repensar la regla del donante fallecido han sugerido que lo que justifica la extracción de órganos de pacientes que han perdido la mayoría de sus funciones encefálicas no es la pretensión de que esos pacientes están realmente muertos, sino otra de carácter moral. Sería según ellos aceptable obviar esa regla cuando el donante, al tener severamente afectado su cerebro, ya no pudiera ser dañado, y solo si hubiera dado por anticipado y de manera explícita su consentimiento para la extracción (Miller, Truog and Brock, 2010). En la práctica, la regla del donante fallecido podría admitir excepciones en aquellos casos en los que el paciente se encuentre más allá de todo daño posible. ${ }^{24}$ La muerte cerebral, el estado vegetativo permanente y la anencefalia son tres diagnósticos en los que existen altas probabilidades de que esta circunstancia se cumpla (Council on Ethical and Judicial Affairs, 1995; Machado, 1999; The Multi-Society Task Force on PVS, 1994a; The MultiSociety Task Force on PVS, 1994b). ${ }^{25}$ Con la excepción del diagnóstico de anencefalia, en el que no se puede obtener el consentimiento previo del donante - lo cual exigiría obtener una autorización de los responsables legales - en los demás casos la donación sería aceptable si el propio paciente hubiera dado su consentimiento explícito. Las nociones morales de daño y de consentimiento informado forman parte del tipo apropiado de argumento para justificar las políticas de trasplante de órganos al plantear con franqueza el debate en términos morales. Resulta esto más conveniente que hacerlo de acuerdo a una distinción de pretendido carácter natural, aparentemente sólida, pero conceptualmente ruinosa. Por otro lado, se evita de este modo recurrir a criterios arbitrarios, como lo "socialmente aceptado", para distinguir a los muertos de los vivos. 


\section{CONCLUSIONES Y PREGUNTAS NO RESPONDIDAS}

La propuesta que he defendido en este artículo asume el carácter borroso e incierto del estatus vital de los pacientes en muerte cerebral a la vez que reconoce el valor moral y político del pluralismo. Sin embargo, deja abierta la cuestión sobre el alcance y los límites de ese pluralismo. Habilita un espacio para el pluralismo en lo concerniente a la decisión sobre la extracción de órganos, pero niega la oportunidad de que la línea que traza una separación entre la vida y la muerte se decida por consideraciones morales camufladas con un discurso científico. Al mismo tiempo, reconoce que la muerte tiene un carácter gradual, que la muerte encefálica guarda cierto parecido de familia con el paradigma de la muerte y que su diagnóstico depende tanto de juicios descriptivos como de juicios normativos. Esto puede parecer contradictorio. En realidad, mi propuesta deja sin responder la pregunta de cuándo alguien ha muerto $y$, si bien me he pronunciado en contra de la asimilación de la muerte encefálica a la muerte humana, solo lo he hecho sobre la base de los intentos fracasados de establecer tal ecuación a partir de premisas biológicas. En último término, se sugiere qué no es la muerte, pero no se señala qué condición sí lo es. La cuestión sobre el diagnóstico queda pospuesta ante una reconocida incapacidad por establecer objetivamente una frontera dicotómica en el continuo de degradación celular y pérdida progresiva de funciones en que consiste el morir.

Mi propuesta sí ofrece, en cambio, una justificación moral de la extracción de órganos, basada en las nociones de daño y de consentimiento. Diferenciar lo permitido de lo prohibido sobre la base de un criterio moral, evidentemente, plantea riesgos y retos. Una posible objeción a este planteamiento es que todo fundamento moral resulta tan endeble como uno de tipo pseudocientífico. Sin embargo, la fragilidad de este tipo de propuestas es al menos lo suficientemente explícita como para evitar que el debate se cierre antes de que haya tenido lugar.

\section{AGRADECIMIENTOS}

Este artículo ha sido escrito con apoyo del proyecto KONTUZ! (Plan Nacional de I+D+i, Subprograma de Proyectos de Investigación Fundamental no Orientada - FFI2011-24414).
1 Desde la teoría de la termodinámica Van Hooft estima que "un organismo vivo es un sistema negentrópico que realiza intercambios metabólicos y de información con su ambiente" (VanHooft, 2004, 148)

2 REAL DECRETO 2070/1999, de 30 de diciembre, Artículo 10. Extracción de órganos de fallecidos: condiciones y requisitos.

3 Ibid.

4 Se trata de una decisión sobre aquello que es esencial para la vida, cuya pérdida constituye por tanto la muerte de la persona. Tal decisión justifica por qué alguien es considerado muerto a pesar de que persistan en su organismo otras actividades (Gervais, 1986, 2-5)

5 Pollice verso es el gesto con el puño cerrado y el pulgar hacia abajo con el que el público del circo romano expresaba su deseo de que se diera muerte a un gladiador.

6 La isquemia es la degradación celular resultante a la falta de oxígeno en órganos y tejidos.
7 Según Bernat y sus colaboradores, las implicaciones de ese concepto supuestamente común de la muerte (supuestamente, porque ellos no disponían en ese momento de ningún estudio empírico que hubiera avalado ese concepto de la muerte, que ellos atribuían al concepto comúnmente aceptado) son las siguientes:

- Que solo los organismos vivos pueden morir;

- Que los vivos pueden distinguirse con fiabilidad de los muertos;

- Que el momento en que un organismo deja de estar vivo y empieza a estar muerto puede ser identificado;

- Que durante algún tiempo después de la muerte, el cuerpo permanece intacto, e incluso algunas partes del organismo continúan funcionando;

- Que la concepción común de la muerte tiene un carácter sistémico, es decir que en su definición de la muerte, las personas se refieren generalmente a la muerte de un organismo como un todo, o en tanto que conjunto, y no a la muerte de todo el organismo, es decir, a la

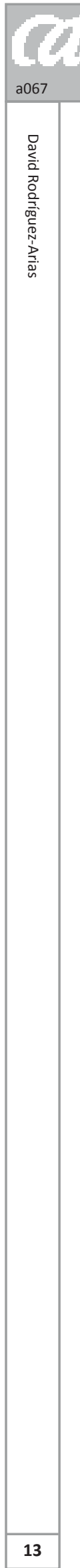

muerte de todos sus tejidos y órganos (death of the organism as a whole vs death of the whole organism), o - señalan los autores - a la interacción de los subsistemas". (Bernat et al., 1981, 390).

8 Widjicks ha mostrado una asombrosa variabilidad entre las legislaciones de diferentes países con respecto a las pruebas requeridas para determinar la muerte cerebral (Wijdicks, 2002).

9 Un debate pormenorizado de todas las propuestas relativas a la muerte cerebral puede encontrarse en (Youngner, Arnold and Schapiro, 1999).

10 Se ha demostrado empíricamente que ciertas funciones integradoras no radican en el cerebro. Por ejemplo la función cardiaca, que depende del sistema nervioso autónomo (Halevy and Brody, 1993).

11 No es necesario que se haya destruido toda actividad en el cerebro para que alguien esté en muerte cerebral. Lo importante, se dice, es que esa actividad no esté organizada (Bernat, 1992). Sin embargo, se ha demostrado que el cerebro de una persona correctamente diagnosti- 
cada en muerte cerebral conserva ciertas funciones integradoras. Más importante aún es que algunas de esas funciones tienen precisamente un papel integrador en el resto del organismo (Halevy and Brody, 1993). Por otro lado, el neurólogo y bioético Alan Shewmon ha identificado que varias funciones integradoras no radican en el cerebro ni están mediadas por él. Este autor menciona una serie de ejemplos de funciones que son predicables del conjunto del organismo, y que, de hecho, son condiciones y consecuencias de su funcionamiento integrado, pero que están presentes en pacientes diagnosticados en muerte cerebral: homeostasis, eliminación, desintoxicación y reciclaje del desgaste celular, equilibrio de la energía (interacción entre el hígado, el sistema endocrino, los músculos y la grasa), cicatrización de las heridas, lucha contra las infecciones (interacción entre el sistema linfático, inmunológico, la médula ósea y el sistema vascular), desarrollo de respuesta febril ante las infecciones, gestación exitosa de un feto en una mujer, maduración sexual en el caso de los niños, crecimiento, respuesta hormonal y cardiovascular en el momento de la incisión sin anestesia durante la extracción de órganos (Shewmon, 2001).

12 Shewmon ha tratado al menos 37 niños en muerte cerebral cuyos padres rechazaban el criterio de muerte cerebral y decidieron mantenerlos conectados a un respirador. Más de la mitad de esos pacientes "sobrevivieron" más de un mes y un tercio más de dos. Siete "sobrevivieron" más de seis meses y cuatro más de un año. El que más tiempo "sobrevivió" lo hizo durante más de 15 años. Por lo tanto, la idea sobre el pronóstico catastrófico a corto plazo en que se basa la concepción naturalista, es una idea errónea (Shewmon, 1998).

13 En Japón, los pacientes en muerte cerebral son por defecto consideradas como vivas, a no ser que el propio paciente hubiera decidido ser considerado como muerto y sus familiares estén de acuerdo (Bagheri, 2007; Lock, 1999).

14 A los individuos competentes se les reconoce un derecho a elegir el trato que la medicina les debe ofrecer una vez que hayan perdido su capacidad para tomar decisiones. La ley básica 41/2002 reguladora de la autonomía del paciente da cuenta de ello a través de las instrucciones previas. También se reconoce el derecho a aceptar la donación de órganos.

15 Este tema lo ha abordado sobre todo en (Putnam, 2004). Putnam atribuye el origen de la dicotomía a la crítica que
Hume hace de la inferencia que lleva del es al debe; y a positivistas lógicos como Carnap (Capítulo I).

16 Putnam sitúa el origen de esta creencia en la concepción de la verdad como representación o copia (lo que Rorty dio en Ilamar el Espejo de la Naturaleza). El argumento sería el siguiente: si lo verdadero solo puede ser copia o representación, y los valores no representan nada, los valores no pueden ser objetivos y no se puede decir de los enunciados morales que sean verdaderos ni falsos (Putnam, 1981).

17 Estos ejemplos pueden encontrarse en (Baggini and Fosl, 2007, 119 y ss).

18 Para desmantelar la dicotomía hechosvalores, Putnam se ocupa más de demostrar que los enunciados científicos incorporan valores -en particular valores epistémicos- que de demostrar que los enunciados morales son objetivos.

19 Lo que justificaría el criterio cardiorrespiratorio y el criterio de muerte encefálica (whole brain death), es que solo en esos casos se da una pérdida irreversible del funcionamiento integrado del organismo como conjunto; lo que justificaría el criterio de muerte cortical (higher brain) es que las funciones corticales son necesarias y suficientes para conservar lo esencial de la vida humana (o para conservar lo que es moralmente esencial para la vida humana), a saber, la conciencia y la cognición.

20 J. Botkin y S. Post citan a Ladd como el primero en haber intentado entender la muerte cerebral sin hacerlo a partir de una definición que reuniera las condiciones necesarias y suficientes (Botkin and Post, 1992). Más recientemente, W. Chiong, ha reabierto esta vía como posible explicación de la muerte cerebral (Chiong, 2005).

21 El concepto de "droga" aglutina un conjunto tan heterogéneo de sustancias que es difícil encontrar un solo rasgo que todas tengan en común: algunas son legales y otras no lo son, algunas producen un efecto relajante y otras un efecto excitante, algunas agudizan la percepción y otras la distorsionan, algunas pueden matar y otras no, algunas crean adicción y otras no.

22 El gobierno francés se ha enfrentado a problemas similares de definición cuando ha querido prohibir las "sectas". En el informe de expertos que pidió el gobierno francés, se mostró que las organizaciones religiosas sospechosas de merecer el calificativo de secta eran de naturaleza, métodos, objetivos y dimensiones muy variables. Por otro lado, se comprobó que muchas organizaciones religiosas de reconocido prestigio compartían algunos rasgos supuestamente característicos de las agrupaciones sectarias. La dificultad con la que se enfrentó el gobierno francés fue ofrecer una definición de secta que incluyera a todas las organizaciones perniciosas y solo a ellas. El informe presentado al Presidente de la República sobre la Misión interministerial de vigilancia y lucha contra las derivas sectarias puede consultarse en http:// www.ladocumentationfracaise. $\mathrm{fr} / \mathrm{ra}$ pports-publics/134000269/index.shtml $(19 / 07 / 2013)$

23 Este estado se caracteriza por un daño del tronco cerebral que impide la comunicación y el movimiento de todo el cuerpo, pero que es compatible con la permanencia de la conciencia. En español, se traduce como "síndrome de encarcelamiento" (Allen, 1993).

24 Entiendo por daño toda carencia severa que implique un debilitamiento necesario del bienestar y de la satisfacción de una necesidad básica para un individuo determinado: alimento, agua, cobijo, y una protección básica de salud. Alguien se encontraría más allá de todo daño posible cuando hubiese perdido de forma definitiva la capacidad de experimentar en primera persona carencias de ese tipo. También cuando, a pesar de conservar la capacidad para experimentar un daño, hubiese consentido libremente a que se le anestesiara para no percibirlo.

25 A pesar de las reiteradas propuestas que ha habido para integrar tanto a pacientes en estado vegetativo como a bebés anencefálicos en la categoría de donantes potenciales (Hoffenberg et al., 1997; Truog and Fletcher, 1990), siguen persistiendo dudas al respecto, motivadas en gran medida por la sospecha de que el aumento de órganos disponibles gracias a esos pacientes no sería lo suficientemente importante como para justificar el posible riesgo que un necesario cambio en la definición y los criterios para determinar la muerte podría acarrear para la sociedad (Shewmon, Capron, Peacock and Schulman, 1989). Verheijde y otros se refieren a diferentes estudios que sugieren la ausencia de certeza sobre la supuesta pérdida total y definitiva de conciencia en los individuos en muerte encefálica, lo que implicaría que incluso esos individuos podrían requerir anestesia en el momento de la extracción (Verheijde and Rady, 2011). 
Ad Hoc Committee of the Harvard Medical School to Examine the Definition of Brain Death (1968). "A definition of irreversible coma. Report of the Ad Hoc Committee of the Harvard Medical School to Examine the Definition of Brain Death". Jama, 205 (6), pp. 337-340.

Allen, C. M. (1993). "Conscious but paralysed: releasing the locked-in". Lancet, 342 (8864), pp. 130-131.

Arnold, R. M. and Youngner, S. J. (1993). "The dead donor rule: should we stretch it, bend it, or abandon it?". Kennedy Inst Ethics J, 3 (2), pp. 263-278.

Baggini, J. and Fosl, P. S. (2007). The ethics toolkit. Cambridge: Blackwell.

Bagheri, A. (2007). "Individual choice in the definition of death". J Med Ethics, 33 (3), pp.146-149.

Bernat J. L. (2010). "Brain death is a scientific concept". Lancet; 375 (9714), 554; author reply.

Bernat J. L. (2013). “Determining Death in Uncontrolled DCDD Organ Donors". The Hastings Center report; 43 (1), 30-3. Epub 2013/01/15.

Bernat J. L. (1992). "How much of the brain must die in brain death?". The Journal of clinical ethics; 3 (1), 21-6; discussion 7-8.

Bernat, J. L. (1999). "Refinements in the definition and criterion of death". En S. J. Youngner, R. Arnold and R. Schapiro, The definition of death: contemporary controversies. Baltimore: The Johns Hopkins University Press, pp. 83-92.

Bernat, J. L.; Culver, C. M. et al. (1981). “On the definition and criterion of death". Ann Intern Med, 94 (3), pp. 389-394.

Botkin, J. R. and Post, S. G. (1992). "Confusion in the determination of death: distinguishing philosophy from physiology". Perspect Biol Med, 36 (1), pp. 129-138.

Capron, A. M. (1999). "The bifurcated legal standard for determining death: does it work?". En S. J. Youngner, R. Arnold and R. Schapiro, The definition of death: contemporary controversies. Baltimore: The Johns Hopkins University Press, pp. 117-136.

Council on Ethical and Judicial Affairs AMA (1995). "The use of anencephalic neonates as organ donors". JAMA: the journal of the American Medical Association, 273 (20), 1614-8.
Chiong, W. (2005). "Brain death without definitions". Hastings Cent Rep, 35 (6), pp. 20-30.

Escalante, J. (1996). "La definición de la muerte". En J. Gafo (ed.), Trasplante de órganos: problemas técnicos, éticos y legales. Madrid: Universidad Pontificia de Comillas, pp. 53-74.

Gervais, K. (1986). Redefining Death. New Haven: Yale University Press

Gomila, A. (1999). Cómo se viene la muerte. Universidad de la Laguna, Volumen extraordinario, pp. 337347.

Green, M. B. and Wikler, D. (1980). "Brain death and personal identity". Philos Public Aff, 9 (2), pp. 105-133.

Halevy, A. and Brody, B. (1993). "Brain death: reconciling definitions, criteria, and tests". Ann Intern Med, 119 (6), pp. 519-525.

Herpin, N. and Paterson, F. (2000). "Le don d'organes et la perception de la mort par les Français: les systémistes et les intégralistes". En R. Carvais and M. Sasportes, La greffe humaine. Paris: PUF, pp. 789-814.

Hoffenberg, R.; Lock, M.; Tilney, N; Casabona, C; Daar, A. S.; Guttmann, R. D. et al. (1997). "Should organs from patients in permanent vegetative state be used for transplantation? International Forum for Transplant Ethics". Lancet; 350 (9087), 1320-1.

Lane, A.; Westbrook, A. et al. (2004). "Maternal brain death: medical, ethical and legal issues". Intensive Care Med, 30 (7), pp. 1484-1486.

Lock, M. (1999). "The problem of brain death: Japenese disputes about bodies and modernity". En S. J. Youngner, R. Arnold and R. Schapiro, The definition of death: contemporary controversies. Baltimore: The Johns Hopkins University Press, pp. 239-256.

Lock, M. (2002). Twice Dead. Organ transplants and the reinvention of death. London: University of California Press.

Machado C. (1999). "Consciousness as a definition of death: its appeal and complexity". Clin Electroencephalogr; 30 (4), 156-64.

Machado, C. (2005). "The first organ transplant from a brain-dead donor". Neurology 64 (11), pp. 1938-1942.
Molina, A.; Rodríguez-Arias, D. et al. (2008). "Should individuals choose their definition of death?". Journal of medical ethics, 34 (9), pp. 688-9.

Mollaret, P. and Goulon, M. (1959). "Le coma dépassé”. Rev Neurol (Paris), 101, pp. 3-15.

Munjal, K. G.; Wall, S. P.; Goldfrank, L. R.; Gilbert, A.; Kaufman, B. J. and Dubler, N. N. (2013). "A rationale in support of uncontrolled donation after circulatory determination of death". The Hastings Center report, 43 (1), 19-26. Epub 2012/12/21.

Nelson, H. L. (1994). "The architect and the bee: some reflections on postmortem pregnancy". Bioethics, 8 (3), pp. 247-267.

President's Commission for the Study of Ethical Problems in Medicine and Biomedical and Behavioral Research (1981). "Guidelines for the determination of death. Report of the medical consultants on the diagnosis of death to the President's Commission for the Study of Ethical Problems in Medicine and Biomedical and Behavioral Research". Jama, 246 (19), pp. 2184-2186.

Putnam, H. (1981). "Hecho y valor". En Razón, verdad e historia. Tecnos: Madrid, pp. 132-152.

Putnam, H. (2004). El desplome de la dicotomía hecho-valor y otros ensayos. Barcelona: Paidós.

Robertson, J. A. (1999). "The dead donor rule”. Hastings Cent Rep, 29 (6), pp. 6-14.

Rodríguez-Arias, D.; Molina, A. (2007). "Pluralismo en torno al significado de la muerte cerebral y/o revisión de la regla del donante fallecido". Laguna, 21, pp. 65-80.

Rodríguez-Arias, D.; Deballon, I. O. (2012). "Protocols for uncontrolled donation after circulatory death". Lancet, 379 (9823), 1275-6. Epub 2012/04/10.

Rorty, R. (2000). El pragmatismo, una versión. Barcelona: Ariel.

Schwartz, P. H. (2007). "Decision and discovery in defining 'disease'". En H. Kincaid and J. McKitrick, Establishing medical reality. Essays in the metaphysicis and epistemology of biomedical science. Dordrecht: Springer, pp. 47-63.

Shewmon, A. D. (2001). "The brain and somatic integration: insights into the standard biological rationale for equating "brain death" with death". J Med Philos, 26 (5), pp. 457-78. 
Shewmon, A. D. (1998). "Chronic 'brain death': meta-analysis and conceptual consequences". Neurology 51 (6), pp. 1538-1545.

Shewmon D. A.; Capron, A. M.; Peacock, W. J.; Schulman, B. L. (1989). "The use of anencephalic infants as organ sources. A critique". JAMA: the journal of the American Medical Association; 261 (12), 1773-81.

Singer, P. (1997). Repensar la vida y la muerte. El derrumbe de nuestra ética tradicional. Barcelona: Paidós.

Siegler, M. and Wikler, D. (1982). "Brain death and live birth". Jama, 248 (9), pp. 1101-1102.

Siminoff, L. A.; Burant, C. et al. (2004). "Death and organ procurement: public beliefs and attitudes". Soc Sci Med, 59 (11), pp. 2325-2334.

Slettedal, J. K.; Lyberg, T. et al. (2007). "Regeneration of the epithelium in organcultured donor corneas with extended post-mortem time". Acta Ophthalmol Scand, 85 (4), pp. 371-376.

Spike, J. (1999). "Brain death, pregnancy, and posthumous motherhood". J Clin Ethics, 10 (1), pp. 57-65.
Stoecker, R. (2012). "Der Tod als Voraussetzung der Organspende?". Zeitschrift für medizinische Ethik, 58. Jahrgang 2012 Heft2, 99-116.

The Multi-Society Task Force on PVS. (1994a). "Medical aspects of the persistent vegetative state (1)". The New England journal of medicine, 330 (21), 1499-508.

The Multi-Society Task Force on PVS. (1994b). "Medical aspects of the persistent vegetative state (2)". The New England journal of medicine, 330 (22), 1572-9.

Truog, R. D.; Fletcher, J. C. (1990). "Brain death and the anencephalic newborn". Bioethics; 4 (3), 199-215.

Van-Hooft, S. (2004). Life, Death, and Subjectivity: Moral Sources in Bioethics. Amsterdam: Value Inquiry Book Series.

Veatch, R. M. (1999). "The conscience clause: how much individual choice in defining death can our society tolerate?". En S. J. Youngner, R. Arnold and R. Schapiro, The definition of death: contemporary controversies. Baltimore: The Johns Hopkins University Press, pp. 137-160.
Verheijde, J. L.; Rady, M. Y. (2011). "Justifying physician-assisted death in organ donation". The American journal of bioethics: $A J O B, 11$ (8), 52-4. 08/03.

Wijdicks, E. F. (2002). "Brain death worldwide: accepted fact but no global consensus in diagnostic criteria". Neurology, 58 (1), pp. 20-25

Wittgenstein, L. (2004). Investigaciones filosóficas. Barcelona: Crítica.

Youngner, S. J.; Arnold, R. et al. (1999). The definition of death: contemporary controversies. Baltimore: The Johns Hopkins University Press.

Youngner, S. J.; Arnold, R. M. et al. (1999). "When is 'dead'?". Hastings Cent Rep, 29 (6), pp. 14-21.

Youngner, S. J. and Arnold, R. M. (2001). "Philosophical debates about the definition of death: who cares?". J Med Philos, 26 (5), pp. 527-37.

Youngner, S. J.; Landefeld, C. S.; Coulton, C. J.; Juknialis, B. W.; Leary, M. (1989). "'Brain death' and organ retrieval. A cross-sectional survey of knowledge and concepts among health professionals". JAMA : the journal of the American Medical Association, 261 (15), 2205-10. 